EUROPEAN JOURNAL OF PURE AND APPLIED MATHEMATICS

Vol. 15, No. 1, 2022, 158-168

ISSN 1307-5543 - ejpam.com

Published by New York Business Global

\title{
A Note on the Infinite Sum of the Lerch function
}

\author{
Robert Reynolds ${ }^{1, *}$, Allan Stauffer ${ }^{1}$ \\ ${ }^{1}$ Department of Mathematics and Statistics, Faculty of Science, York University, Toronto, \\ Ontario, Canada, MЗJ1P3
}

\begin{abstract}
We derive the infinite sum of the Lerch function in terms of the incomplete gamma function and the Lerch function. Special cases are evaluated in terms of fundamentals constants. All the results in this work are new.
\end{abstract}

2020 Mathematics Subject Classifications: 30E20, 33-01, 33-03, 33-04, 33-33B

Key Words and Phrases: Lerch function, incomplete gamma function, Catalan's constant, Apréy's constant

\section{Introduction}

Infinite sums of special functions have been studied in the works of $[1,5,6,10,13]$. In this present work we derive a new expression for the Lerch function in terms of the infinite sum of the Incomplete gamma function given by

$$
\begin{aligned}
& \sum_{n=1}^{\infty} 2^{k-n+1}\left(2^{-n}\right)^{k} \\
& e^{m 2^{1-n}} \Phi\left(-e^{2^{1-n} m},-k, 2^{n-1} \log (a)+1\right) \\
& =-\frac{a^{-m}(-m)^{-k} \Gamma(k+1,-m \log (a))}{m}-2^{k+1} e^{2 m} \Phi\left(e^{2 m},-k, \frac{\log (a)}{2}+1\right)
\end{aligned}
$$

where the variables $k, a, m$ are general complex numbers. This new expression is then used to derive special cases in terms of fundamental constant and special functions. The derivations follow the method used by us in [12]. This method involves using a form of the generalized Cauchy's integral formula given by

$$
\frac{y^{k}}{\Gamma(k+1)}=\frac{1}{2 \pi i} \int_{C} \frac{e^{w y}}{w^{k+1}} d w
$$

*Corresponding author.

DOI: https://doi.org/10.29020/nybg.ejpam.v15i1.4137

Email addresses: milver@my.yorku.ca (R. Reynolds), stauffer@yorku.ca (A. Stauffer) 
where $y, w \in \mathbb{C}$ and $C$ is in general an open contour in the complex plane where the bilinear concomitant [12] has the same value at the end points of the contour. This method involves using a form of equation (2) then multiplies both sides by a function, then takes the definite integral of both sides. This yields a definite integral in terms of a contour integral. Then we multiply both sides of equation (2) by another function and take the infinite sum of both sides such that the contour integral of both equations are the same.

\subsection{The Incomplete Gamma Function}

The multivalued incomplete gamma functions [3], $\gamma(s, z)$ and $\Gamma(s, z)$, are defined by

$$
\gamma(s, z)=\int_{0}^{z} t^{s-1} e^{-t} d t
$$

and

$$
\Gamma(s, z)=\int_{z}^{\infty} t^{s-1} e^{-t} d t
$$

where $\operatorname{Re}(z)>0$. The incomplete gamma function has a recurrence relation given by

$$
\gamma(s, z)+\Gamma(s, z)=\Gamma(s),
$$

where $z \neq 0,-1,-2, \ldots$ The incomplete gamma function is continued analytically by

$$
\gamma\left(a, z e^{2 m \pi i}\right)=e^{2 \pi m i a} \gamma(a, z)
$$

and

$$
\Gamma\left(s, z e^{2 m \pi i}\right)=e^{2 \pi m i s} \Gamma(s, z)+\left(1-e^{2 \pi m i s}\right) \Gamma(s),
$$

where $m \in \mathbb{Z}$. When $z \neq 0, \Gamma(s, z)$ is an entire function of $s$ and $\gamma(s, z)$ is meromorphic with simple poles at $s=-n$ for $n=0,1,2, \ldots$ with residue $\frac{(-1)^{n}}{n !}$. These definitions are listed in Section 8.2(i) and (ii) in [3].

\section{The Lerch function}

We use equation (1.11.3) in [2] where $\Phi(z, s, v)$ is the Lerch function which is a generalization of the Hurwitz zeta $\zeta(s, v)$ and Polylogarithm functions $L i_{n}(z)$. The Lerch function has a series representation given by

$$
\Phi(z, s, v)=\sum_{n=0}^{\infty}(v+n)^{-s} z^{n}
$$

where $|z|<1, v \neq 0,-1,-2,-3, . .$, and is continued analytically by its integral representation given by

$$
\Phi(z, s, v)=\frac{1}{\Gamma(s)} \int_{0}^{\infty} \frac{t^{s-1} e^{-v t}}{1-z e^{-t}} d t=\frac{1}{\Gamma(s)} \int_{0}^{\infty} \frac{t^{s-1} e^{-(v-1) t}}{e^{t}-z} d t
$$

where $\operatorname{Re}(v)>0$, and either $|z| \leq 1, z \neq 1, \operatorname{Re}(s)>0$, or $z=1, \operatorname{Re}(s)>1$. 


\section{Contour integral representation for the infinite sum of the Lerch function}

We use the method in [12]. The cut and contour are in the first quadrant of the complex $w$-plane with $0<R e(w+m)<1$. The cut approaches the origin from the interior of the first quadrant and goes to infinity vertically and the contour goes round the origin with zero radius and is on opposite sides of the cut. Using a generalization of Cauchy's integral formula (2) we first replace $y \rightarrow \log (a)+2^{1-n}(y+1)$ then multiply both sides by $(-1)^{y} e^{m 2^{1-n}(y+1)}$ and take the infinite sums over

$y \in[0, \infty)$ and $n \in[1, \infty)$ and simplify in terms of the Lerch function to get

$$
\begin{aligned}
& \sum_{n=1}^{\infty} \frac{2^{k-n+1}\left(2^{-n}\right)^{k} e^{m 2^{1-n}} \Phi\left(-e^{2^{1-n} m},-k, 2^{n-1} \log (a)+1\right)}{\Gamma(k+1)} \\
& =\frac{1}{2 \pi i} \sum_{n=1}^{\infty} \sum_{y=0}^{\infty} \int_{C} 2(-1)^{y} a^{w} w^{-k-1} e^{2^{1-n}(y+1)(m+w)} d w \\
& =\frac{1}{2 \pi i} \int_{C} \sum_{n=1}^{\infty} \sum_{y=0}^{\infty} 2(-1)^{y} a^{w} w^{-k-1} e^{2^{1-n}(y+1)(m+w)} d w \\
& =\frac{1}{2 \pi i} \int_{C} \sum_{n=1}^{\infty}\left(2^{-n} a^{w} w^{-k-1} \tanh \left(2^{-n}(m+w)\right)+2^{-n} a^{w} w^{-k-1}\right) d w \\
& =\frac{1}{2 \pi i} \int_{C}\left(a^{w} w^{-k-1} \operatorname{coth}(m+w)-\frac{a^{w} w^{-k-1}}{m+w}+a^{w} w^{-k-1}\right) d w
\end{aligned}
$$

from equations (5.3.8.4) in [11] and (1.232.1) in [4] where $\operatorname{Re}(w+m)>0$ and $\operatorname{Im}(w+m)>$ 0 in order for the sums to converge.

\subsection{Additional contour integral}

Using a generalization of Cauchy's integral formula (2) we first replace $y \rightarrow \log (a)$ then multiply both sides by $2^{-n}$ and take the infinite sum over $n \in[1, \infty)$ to get

$$
\begin{aligned}
-\sum_{n=1}^{\infty} \frac{2^{-n} \log ^{k}(a)}{\Gamma(k+1)} & =-\frac{1}{2 \pi i} \sum_{n=1}^{\infty} \int_{C} 2^{-n} a^{w} w^{-k-1} d w \\
& =-\frac{1}{2 \pi i} \int_{C} \sum_{n=1}^{\infty} 2^{-n} a^{w} w^{-k-1} d w \\
& =-\frac{1}{2 \pi i} \int_{C} a^{w} w^{-k-1} d w
\end{aligned}
$$


4. Contour integral representation for the Lerch and incomplete gamma functions

Using a generalization of Cauchy's integral formula (2) we first replace $y \rightarrow \log (a)+$ $2(y+1)$ then multiply both sides by $e^{2 m(y+1)}$ and take the infinite sum over $y \in[0, \infty)$ and simplify in terms of the Lerch function to get

$$
-\frac{2^{k+1} e^{2 m} \Phi\left(e^{2 m},-k, \frac{\log (a)}{2}+1\right)}{\Gamma(k+1)}=\frac{1}{2 \pi i} \int_{C} a^{w} w^{-k-1} \operatorname{coth}(m+w)+a^{w} w^{-k-1} d w
$$

from equation (1.232.1) in [4] where $\operatorname{Im}(w+m)>0$ in order for the sum to converge.

\subsection{Additional contour integral}

Using a generalization of Cauchy's integral formula (2) we first replace $y \rightarrow \log (a)$ and simplify to get

$$
\frac{\log ^{k}(a)}{\Gamma(k+1)}=\frac{1}{2 \pi i} \int_{C} a^{w} w^{-k-1} d w
$$

\subsection{Contour integral representation for the incomplete gamma function}

Using a generalization of Cauchy's integral formula (2) we first replace $y \rightarrow y+\log (a)$ then multiply both sides by $e^{m y}$ and take the definite integral over $y \in[0, \infty)$ to get

$$
\frac{a^{-m}(-m)^{-k-1} \Gamma(k+1,-m \log (a))}{\Gamma(k+1)}=-\frac{1}{2 \pi i} \int_{C} \frac{a^{w} w^{-k-1}}{m+w} d w
$$

from equation (3.383.4) in [4] where $0<\operatorname{Re}(w+m)<1$.

\section{Infinite sum of the Lerch function in terms of the Lerch function}

Theorem 1. For all $k, a, m \in \mathbb{C}$,

$$
\begin{aligned}
\sum_{n=1}^{\infty} 2^{k-n+1}\left(2^{-n}\right)^{k} e^{m 2^{1-n}} \Phi\left(-e^{2^{1-n} m},-k, 2^{n-1} \log (a)+1\right) & \\
= & -\frac{a^{-m}(-m)^{-k} \Gamma(k+1,-m \log (a))}{m}-2^{k+1} e^{2 m} \Phi\left(e^{2 m},-k, \frac{\log (a)}{2}+1\right)
\end{aligned}
$$

or

$$
\begin{aligned}
& \sum_{n=1}^{\infty} 2^{k-n+1}\left(2^{-n}\right)^{k} m^{2^{-n}} \Phi\left(-m^{2^{-n}},-k, 2^{n}(a-1)+1\right) \\
& \quad=-2^{k+1} m \Phi(m,-k, a)-\frac{2^{k+1}\left(e^{2(a-1)}\right)^{-\frac{\log (m)}{2}}(-\log (m))^{-k} \Gamma(k+1,(1-a) \log (m))}{\log (m)}
\end{aligned}
$$


Proof. Observe that the the addition of the right-hand sides of equations (5) and (6) is equal to the addition of the right-hand sides of equation (7), (8) and (9) so we may equate the left-hand sides and simplify the the Gamma function to yield the stated result.

\section{Special cases}

In this section we will evaluate equation (10) for various parameter ranges. We will use the following functions for simplification; exponential integral function $E_{n}(z)$ given in section (8.19) in [3], and the exponential integral function $E_{i}(z)$ given in equation (6.11.2) [3], and the Riemann zeta function given in equation (25.2.1) in [3].

Example 1. The degenerate case.

$$
\sum_{n=1}^{\infty} 2^{1-n}\left(1-\frac{1}{e^{m 2^{1-n}}+1}\right)=\sum_{n=1}^{\infty} 2^{-n}\left(\tanh \left(m 2^{-n}\right)+1\right)=-\frac{1}{m}+\operatorname{coth}(m)+1
$$

Proof. Use equation (10) and set $k=0$ and simplify using entry (4) in table below $(64: 12: 7)$ in $[9]$.

Example 2. Apéry's constant $\zeta(3)$

$$
\sum_{n=1}^{\infty}(-1)^{2^{-n}} 4^{n-1} \Phi\left((-1)^{1+2^{-n}}, 3,1+2^{n}\right)=\frac{1}{16}\left(-4 E_{3}(-i \pi)-3 \zeta(3)+4\right)
$$

Proof. Use equation (11) and set $m=-1$ and simplify in terms of the Hurwitz zeta function $\zeta(s, a)$ using entry (4) in table below (64:12:7) in [9]. Next set $k=-3, a=2$ and simplify using equation (8.19.2) in [3] and entry (2) in table below (64:7) in [9].

Example 3. Fundamental constant $\zeta(5)$

$$
\sum_{n=1}^{\infty}(-1)^{2^{-n}} 16^{n-1} \Phi\left((-1)^{1+2^{-n}}, 5,1+2^{n}\right)=\frac{1}{256}\left(-16 E_{5}(-i \pi)-15 \zeta(5)+16\right)
$$

Proof. Use equation (11) and set $m=-1$ and simplify in terms of the Hurwitz zeta function $\zeta(s, a)$ using entry (4) in table below (64:12:7) in [9]. Next set $k=-5, a=2$ and simplify using equation (8.19.2) in [3] and entry (2) in table below (64:7) in [9].

Example 4. Catalan's constant $C$

$$
\sum_{n=1}^{\infty}(-1)^{2^{-n}} 2^{n-1} \Phi\left((-1)^{1+2^{-n}}, 2,1+2^{n-1}\right)=-2 C+3+\frac{1}{2} \pi \Gamma\left(0,-\frac{i \pi}{2}\right)
$$


Proof. Use equation (11) and set $m=-1$ and simplify in terms of the Hurwitz zeta function $\zeta(s, a)$ using entry (4) in table below (64:12:7) in [9]. Next set $k=-2, a=3 / 2$ and simplify using equation (8.19.2) in [3], equation (64:4:1) in [9] and equation (2.2.1.2.7) in $[7]$.

Example 5. The exponential integral function $E_{n}(z)$

$$
\sum_{n=1}^{\infty}(-1)^{2^{-n}} \Phi\left((-1)^{1+2^{-n}}, 1,1+2^{n-1}\right)=\frac{1}{2}\left(2 i E i\left(\frac{i \pi}{2}\right)+4+\pi\right)
$$

Proof. Use equation (11) and set $m=-1$ and simplify in terms of the Hurwitz zeta function $\zeta(s, a)$ using entry (4) in table below (64:12:7) in [9]. Next we apply l'Hopital's rule as $k \rightarrow-1$ and simplify using equation (8.19.2) in [3] and equation (64:4:1) in [9].

\section{Example 6.}

$$
\begin{aligned}
\sum_{n=1}^{\infty}(-1)^{2^{-n}}\left(2^{-n}\right)^{3 / 2} \Phi\left((-1)^{1+2^{-n}},-\frac{1}{2},\right. & \left.1+2^{n}\right) \\
& =-E_{-\frac{1}{2}}(-i \pi)+(2 \sqrt{2}-1) \zeta\left(-\frac{1}{2}\right)+1
\end{aligned}
$$

Proof. Use equation (11) and set $m=-1$ and simplify in terms of the Hurwitz zeta function $\zeta(s, a)$ using entry (4) in table below (64:12:7) in [9]. Next set $a=2$ and simplify in terms of the Riemann zeta function $\zeta(s)$ using entry (2) in table below (64:7) in [9]. Next set $k=-1 / 2$ and simplify.

\section{Example 7.}

$$
\sum_{n=1}^{\infty}(-1)^{2^{-n}} \sqrt{2^{-n}} \Phi\left((-1)^{1+2^{-n}}, \frac{1}{2}, 1+2^{n}\right)=-E_{\frac{1}{2}}(-i \pi)+(\sqrt{2}-1) \zeta\left(\frac{1}{2}\right)+1
$$

Proof. Use equation (11) and set $m=-1$ and simplify in terms of the Hurwitz zeta function $\zeta(s, a)$ using entry (4) in table below (64:12:7) in [9]. Next set $a=2$ and simplify in terms of the Riemann zeta function $\zeta(s)$ using entry (2) in table below (64:7) in [9]. Next set $k=1 / 2$ and simplify.

\section{Infinite sum of the Lerch function in terms of the Lerch transformation}

Theorem 2. For $\operatorname{Re}(s)<0,0<v \leq 1,0 \leq \operatorname{Im}(\alpha)<2 \pi$,

$$
\sum_{n=1}^{\infty} e^{\alpha\left(-2^{-n}\right)} 2^{-n-s+1}\left(2^{-n}\right)^{-s} \Phi\left(-e^{-2^{-n} \alpha}, s, 2^{n}(v-1)+1\right)
$$




$$
\begin{gathered}
=2^{1-s} \alpha^{s-1}\left(e^{2(v-1)}\right)^{\alpha / 2} \Gamma(1-s,(v-1) \alpha)-e^{-\alpha} 2^{1-s}\left(\alpha^{s-1} \Gamma(1-s) e^{\alpha v}\right. \\
\left.-i(2 \pi)^{s-1} \Gamma(1-s) e^{\alpha v}\left(e^{i\left(\frac{\pi s}{2}+2 \pi v\right)} \Phi\left(e^{2 i \pi v}, 1-s, 1-\frac{i \alpha}{2 \pi}\right)-e^{-i\left(\frac{\pi s}{2}+2 \pi v\right)} \Phi\left(e^{-2 i \pi v}, 1-s, \frac{i \alpha}{2 \pi}+1\right)\right)\right)
\end{gathered}
$$

Proof. Use equation (12) in [8] and substitute into equation (11) and simplify.

\section{Extended Vălean Infinite Sums and Infinite Products}

In the book of Vălean [14] section (3.59) has some very interesting infinite sums of trigonometric functions. In this section of the present paper we will expand on the sums published by Vălean using a special case of equation (11) for various values of the parameters involved.

\section{Lemma 1.}

$$
\begin{aligned}
& \sum_{n=1}^{\infty}(-1)^{2^{-n}} 2^{k-n+1}\left(2^{-n}\right)^{k} \Phi\left((-1)^{1+2^{-n}},-k, 2^{n}(a-1)+1\right) \\
= & 2^{k+1}\left(2^{k} \zeta\left(-k, \frac{a}{2}\right)-2^{k} \zeta\left(-k, \frac{a+1}{2}\right)\right)+i\left(e^{2(a-1)}\right)^{-\frac{i \pi}{2}}(-i)^{-k} 2^{k+1} \pi^{-k-1} \Gamma(k+1, i(1-a) \pi)
\end{aligned}
$$

Proof. Use equation (11) and set $m=1$ and simplify using entry (5) in Table below $(64: 12: 7)$ in $[9]$.

\section{Example 8.}

$$
\sum_{n=1}^{\infty} \frac{2^{1-2 n}\left(1+\alpha 2^{n}\left(1+e^{i \pi 2^{-n}}\right)\right)}{\cos \left(\pi 2^{-n}\right)+1}=2 \alpha+4 \alpha^{2}\left(e^{2 \alpha}\right)^{-\frac{i \pi}{2}} E_{-1}(-i \pi \alpha)+1
$$

Proof. Use equation (20) and set $k=1$ and replace $a \rightarrow \alpha+1$ and simplify.

\section{Example 9.}

$$
\sum_{n=1}^{\infty} 8^{-n} \sin ^{4}\left(\pi 2^{-n-1}\right) \csc ^{3}\left(\pi 2^{-n}\right)=\frac{1}{\pi^{3}}
$$

Proof. Use equation (20) and set $k=2, a=1$ and simplify.

\section{Example 10.}

$$
\sum_{n=1}^{\infty} 2^{-4 n}\left(\cos \left(\pi 2^{-n}\right)-2\right) \sec ^{4}\left(\pi 2^{-n-1}\right)=\frac{48}{\pi^{4}}-1
$$


Proof. Use equation (20) and set $k=3, a=1$ and simplify.

Example 11. The polylogarithm function $L_{n}(z)$ and Riemann zeta function $\zeta(s)$

$$
\begin{aligned}
\sum_{n=1}^{\infty}-2^{k-n+1}\left(2^{-n}\right)^{k} L i_{-k} & \left((-1)^{1+2^{-n}}\right) \\
& =2^{k+1}\left(-\left(2^{k+1}-1\right) \zeta(-k)+i(-i)^{-k} \pi^{-k-1} \Gamma(k+1)\right)
\end{aligned}
$$

Proof. Use equation (20) and set $a=1$ and simplify using entry (1) in Table below $(64: 7)$ in $[9]$.

Example 12. The polylogarithm function $L i_{n}(z)$

$$
\sum_{n=1}^{\infty} \sqrt{2^{1-n}} L i_{\frac{1}{2}}\left((-1)^{1+2^{-n}}\right)=-(\sqrt{2}-2) \zeta\left(\frac{1}{2}\right)+(-1-i)
$$

Proof. Use equation (24) and set $k=-1 / 2$ and simplify.

\section{Example 13.}

$$
\sum_{n=1}^{\infty} 4^{-n} \sec ^{2}\left(\pi 2^{-n-1}\right)=1-\frac{4}{\pi^{2}}
$$

Proof. Use equation (10) and set $k=1, a=1, m=\pi i / 2$ and simplify using entry (3) in table below $(64: 12: 7)$ in [9].

\subsection{Definite Integral of Infinite Sum}

In this section we extend upon the results in [14]. We take the definite integral of specific infinite sums in terms of hyperbolic trigonometric functions. These definite integrals result in the sum of the logarithm of hyperbolic trigonometric functions which is equivalent to the infinite product of the logarithm of hyperbolic trigonometric functions.

\section{Example 14.}

Using equation (10) and setting $k=1, a=1, m=y$ and simplifying using entry (3) in table below (64:12:7) in [9] we get

$$
\sum_{n=1}^{\infty} 4^{-n} \operatorname{sech}^{2}\left(2^{-n} y\right)=\frac{1}{y^{2}}-\operatorname{csch}^{2}(y)
$$

Next we take the definite integral over $y \in[x, x / 2]$ to get

$$
\int_{x / 2}^{x}\left(\sum_{n=1}^{\infty} 4^{-n} \operatorname{sech}^{2}\left(2^{-n} y\right)=\frac{1}{y^{2}}-\operatorname{csch}^{2}(y)\right) d y
$$


We reverse the order of integration and summation and again take the definite integral over $x \in[z, z / 2]$ to get

$$
\int_{z / 2}^{z}\left(\sum_{n=1}^{\infty}-2^{-n}\left(\tanh \left(2^{-n-1} x\right)-\tanh \left(2^{-n} x\right)\right)=\frac{1}{x}-\operatorname{coth}\left(\frac{x}{2}\right)+\operatorname{coth}(x)\right) d x
$$

Reversing the order of the sum and integral and simplifying we get

$$
\sum_{n=1}^{\infty} \log \left(\cosh ^{2}\left(2^{-n-2} z\right) \cosh \left(2^{-n} z\right) \operatorname{sech}^{3}\left(2^{-n-1} z\right)\right)=\log \left(\tanh ^{2}\left(\frac{z}{4}\right)+1\right)
$$

whence upon replacing $z \rightarrow x$ we obtain that

$$
\prod_{n=1}^{\infty} \frac{\cosh ^{2}\left(2^{-2-n} x\right) \cosh \left(2^{-n} x\right)}{\cosh ^{3}\left(2^{-1-n} x\right)}=1+\tanh ^{2}\left(\frac{x}{4}\right)
$$

\section{Example 15.}

$$
\prod_{n=1}^{\infty} \cosh \left(2^{-n} x\right)\left(\tanh \left(2^{-n-1} x\right)+1\right) e^{2^{-n} \tanh \left(2^{-n-1} x\right) \operatorname{sech}\left(2^{-n} x\right)}=\frac{1}{2}\left(e^{x}+1\right) e^{\frac{1}{x}-\operatorname{coth}\left(\frac{x}{2}\right)+\operatorname{coth}(x)}
$$

Proof. Use the process in Example (14) with $k=1, a=e, m=x$ and simplify.

\section{Example 16.}

$$
\prod_{n=1}^{\infty} \frac{\cosh ^{7}\left(2^{-1-n} x\right) \cosh ^{8}\left(2^{-3-n} x\right)}{\cosh \left(2^{-n} x\right) \cosh ^{14}\left(2^{-2-n} x\right)}=\frac{\cosh ^{6}\left(\frac{x}{4}\right)}{\cosh ^{8}\left(\frac{x}{8}\right) \cosh \left(\frac{x}{2}\right)}
$$

Proof. Use the process in Example (14) with $k=2, a=1, m=x$ and simplify.

\section{Example 17.}

$$
\prod_{n=1}^{\infty} \cosh \left(2^{-n} x\right)\left(\tanh \left(2^{-n-1} x\right)+1\right)=\frac{1}{2} e^{x / 2} \sinh (x) \operatorname{csch}\left(\frac{x}{2}\right)
$$

Proof. Use the process in Example (14) with $k=1, a=e^{2 a}, m=x$ and simplify.

\section{Example 18.}

$$
\prod_{n=1}^{\infty} \frac{\cosh \left(2^{-n} x\right)}{\cosh \left(2^{-1-n} x\right)}=\frac{\sinh (x)}{2 \sinh \left(\frac{x}{2}\right)}
$$

Proof. Use the process in Example (14) with $k=1, a=e^{a}, m=x$ and simplify.

\section{Example 19.}

$$
\prod_{n=1}^{\infty} \frac{\cosh ^{2}\left(2^{-2-n} x\right) \cosh \left(2^{-n} x\right)}{\cosh ^{3}\left(2^{-1-n} x\right)}=\frac{\cosh \left(\frac{x}{2}\right)}{\cosh ^{2}\left(\frac{x}{4}\right)}
$$

Proof. Use the process in Example (14) with $k=1, a=e^{a}, m=x$ by forming a second equation with replacing $a \rightarrow-a$ and simplify. 


\section{Discussion}

The authors construct an infinite sum of the Lerch function $\Phi(k, a, m)$ in terms of the incomplete gamma function $\Gamma(k, a)$ and the Lerch function, where the parameter constraints are wide. The infinite sum of the Lerch function in terms of the Lerch transformation was also derived. The derivations used fundamental constants and special functions, and the infinite sum allowed for a wide range of the parameters. We checked the outcome numerically using Wolfram Mathematica.

\section{References}

[1] D.H. Bailey and J.M. Borwein. Crandall's computation of the incomplete gamma function and the hurwitz zeta function, with applications to dirichlet l-series. Applied Mathematics and Computation, 268:462-477, 102015.

[2] Harry Bateman. Higher Transcendental Functions V.1. McGraw-Hill, 1953.

[3] Nist digital library of mathematical functions. F. W. J. Olver, A. B. Olde Daalhuis, D. W. Lozier, B. I. Schneider, R. F. Boisvert, C. W. Clark, B. R. Miller, B. V. Saunders, H. S. Cohl, and M. A. McClain, eds.

[4] I. S. Gradshteyn and I. M. Ryzhik. Table of integrals, series, and products. Elsevier/Academic Press, Amsterdam, seventh edition, 2007.

[5] S. Kanemitsu, H. Kumagai, H.M. Srivastava, and M. Yoshimoto. Some integral and asymptotic formulas associated with the hurwitz zeta function. Applied Mathematics and Computation, 154:641-664, 072004.

[6] S Kanemitsu, Y Tanigawa, H Tsukada, and M Yoshimoto. Contributions to the theory of the hurwitz zeta-function. Hardy-Ramanujan Journal, Volume 30, 012007.

[7] Leonard Lewin. Polylogarithms and Associated Functions. North Holland, 1981.

[8] F. Oberhettinger. Note on the lerch zeta function. Pacific Journal of Mathematics, 6(1):117 - 120, 1956.

[9] Keith B. Oldham, Jan Myland, and Jerome Spanier. An Atlas of Functions: with Equator, the Atlas Function Calculator. Springer Science \& Business Media, 072010.

[10] R. B. Paris. The stokes phenomenon associated with the hurwitz zeta function $\zeta(s, a)$. Proceedings of the Royal Society A: Mathematical, Physical and Engineering Sciences, 461:297-304, 012005.

[11] Anatoliǔ Platonovich Prudnikov, Yu A. Brychkov, and Oleg Igorevich Marichev. Integrals and Series: More special functions. Gordon and Breach Science Publishers, 1986. 
[12] Robert Reynolds and Allan Stauffer. A method for evaluating definite integrals in terms of special functions with examples. International Mathematical Forum, 15:235244,2020 .

[13] Robert Reynolds and Allan Stauffer. Infinite sum of the incomplete gamma function expressed in terms of the hurwitz zeta function. Mathematics, 9:1952, 082021.

[14] Cornel Ioan Vălean. (Almost) Impossible Integrals, Sums, and Series. Springer, 05 2019. 